\title{
NR3A Modulates the Outer Vestibule of the "NMDA" Receptor Channel
}

\author{
Akira Wada, ${ }^{1}$ Hiroto Takahashi, ${ }^{1}$ Stuart A. Lipton, ${ }^{1,2}$ and H.-S. Vincent Chen ${ }^{1,3}$ \\ ${ }^{1}$ Center for Neuroscience and Aging, Burnham Institute for Medical Research, and Departments of ${ }^{2}$ Neurosciences and ${ }^{3}$ Cardiology, University of \\ California, San Diego, La Jolla, California 92037
}

\begin{abstract}
Classical NMDA receptors (NMDARs), activated by glycine and glutamate, are heteromultimers comprised of NR1 and NR2 subunits. Coexpression of the novel NR3 family of NMDAR subunits decreases the magnitude of NR1/NR2 receptor-mediated currents or forms glycine-activated channels with the NR1 subunit alone. The second (M2) and third (M3) membrane segments of NR1 and NR2 subunits of classical NMDARs form the core of the channel permeation pathway. Structural information regarding NR1/NR3 channels remains unknown. Using the Xenopus oocyte expression system and the SCAM (substituted cysteine accessibility method), we found that M3 segments of both NR1 and NR3A form a narrow constriction in the outer vestibule of the channel, which prevents passage of externally applied sulfhydryl-specific agents. The most internal reactive residue in each M3 segment is the threonine in the conserved SYTANLAAF motif. These threonines appear to be symmetrically aligned. Several NR3A M3 mutations change the behavior of NR1/NR3A channels. Unlike NR1, however, the M3 segment of NR3A does not undergo extensive molecular rearrangement during channel gating by added glycine. Additionally, in the M2 segment, our data suggest that the amino acid at the asparagine (N) site of NR1, but not NR3A, contributes to the selectivity filter of NR1/3A channels. We therefore conclude that NR3A modulates the NR1/NR3A permeation pathway via a novel mechanism of forming a narrow constriction at the outer channel vestibule. This modified channel vestibule may also explain the dominant-negative effect of the NR3 subunit on channel behavior when coexpressed with NR1 and NR2 subunits.
\end{abstract}

Key words: ligand-gated channel; SCAM; permeability; block; glutamate; size filter

\section{Introduction}

NMDA-type glutamate receptors participate in neuronal development, plasticity, and synaptic transmission in the CNS (Nakanishi, 1992). Excessive activation of NMDA receptors (NMDARs) by glutamate, however, mediates neuronal damage in many neurological disorders (Lipton and Rosenberg, 1994; Choi, 1998). In contrast to AMPA- and kainate-type glutamate receptors, all functional NMDARs are heteromultimers (for review, see Wollmuth and Sobolevsky, 2004). Conventional NMDARs composed of NR1 and NR2A-D subunits require dual agonists, glutamate and glycine, for activation. The activity of the NMDAR-associated channel is modulated by voltage-dependent block of $\mathrm{Mg}^{2+}$ (Mayer et al., 1984; Nowak et al., 1984), and the channel manifests high permeability to $\mathrm{Ca}^{2+}$ (for review, see Dingledine et al., 1999). We recently cloned a third member of the NMDAR family, the NR3 subunits, composed of NR3A and

\footnotetext{
Received June 17, 2006; revised 0ct. 3, 2006; accepted 0ct. 31, 2006.

This work was supported by an American Heart Association Scientist Development Grant and an Early Career Development Award from American College of Cardiology Foundation (H.-S.V.C.) and by National Institutes of Health Grants P01 HD29587 and R01 EY05477 (S.A.L.). We thank Maria Talantova for graphical modeling of the NMDA-gated channel.

Correspondence should be addressed to H.-S. Vincent Chen, M.D., Ph.D., Burnham Institute for Medical Research, 10901 North Torrey Pines Road, La Jolla, CA 92037. E-mail: hsv_chen@burnham.org.

A. Wada's present address: University of Illinois Urbana-Champaign, College of Medicine, Urbana, IL 61801.

H. Takahashi's present address: Howard Hughes Medical Institute, Janelia Farm Research Campus, 19700 Helix Drive, Ashburn, VA 20147.

D0I:10.1523/JNEUROSCI.2552-06.2006

Copyright $\odot 2006$ Society for Neuroscience $\quad$ 0270-6474/06/2613156-11\$15.00/0
}

NR3B. Coexpression of NR3 with NR1 and NR2 subunits decreases NR1/NR2 receptor-mediated current amplitude (Sucher et al., 1995; Nishi et al., 2001), unitary conductance (Das et al., 1998), $\mathrm{Ca}^{2+}$ permeability and $\mathrm{Mg}^{2+}$ sensitivity (Matsuda et al., 2002; Sasaki et al., 2002). The mechanism of this "dominantnegative effect" of NR3 subunits remains elusive. Importantly, the NR3 subunits can form glycine-activated cation channels with NR1 in the absence of NR2 subunits in both oocyte (Chatterton et al., 2002) and mammalian expression systems (PiñaCrespo and Heinemann, 2004). Evidence for physiological expression of NR1/NR3 receptors has been described in primary cerebrocortical neurons (Chatterton et al., 2002). These glycineactivated channels are less permeable to $\mathrm{Ca}^{2+}$ and relatively resistant to blockade by $\mathrm{Mg}^{2+}$ and NMDAR open-channel blockers. Structural information regarding NR1/NR3 channels, however, remains unknown.

Based on several studies using the substituted cysteine accessibility method (SCAM), the ionic conducting pathway of the NMDA-gated channel was shown to consist of an extracellular vestibule and an internal narrow constriction formed by the second membrane (M2) pore loop (for review, see Wollmuth and Sobolevsky, 2004). The asparagine (N) residues in the pore loop at the $\mathrm{N}$ site of NR1 and the $\mathrm{N}+1$ site of NR2 subunits form the selectivity filter that controls ionic selectivity (Kuner et al., 1996). The extracellular vestibule is comprised of residues on the $\mathrm{N}$-terminal side of the M1 segment, the C-terminal part of the M3 segment, and the N-terminal part of the M4 segment (Beck et al., 
1999). In NR1/NR2 receptors, the M3 segments of both subunits contribute to the core and the more superficial part of the extracellular vestibule that precedes the narrow constriction of the channel. The M3 segment undergoes extensive molecular rearrangement during channel gating (Sobolevsky et al., 2002a). These M3 segments are staggered relative to each other in the vertical axis of the channel (Sobolevsky et al., 2002b).

Using the Xenopus oocyte expression system and SCAM analysis, we show that the $\mathrm{N}$-site residue of NR1 but not NR3 subunits forms the tip of the channel pore loop. The M3 segments of both NR1 and NR3A align symmetrically and constitute the core of a narrow extracellular channel vestibule, which precludes passage of external sulfhydryl-specific agents. Our data suggest that NR3A modulates the NR1/NR3A channel via a novel mechanism of modifying the channel outer vestibule.

\section{Materials and Methods}

\section{Site-directed mutagenesis}

Cloned cDNAs for the rat NMDA receptor subunits NR1-1a (pJS1) (Sullivan et al., 1994) and NR3A were used as templates. Amino acids are numbered from the initiator methionine in the NR1 and NR3 clones (Chatterton et al., 2002). The mutant NMDAR subunits used here were all generated in our laboratory. We used the PCR-based QuikChange Site-Directed Mutagenesis kit from Stratagene (La Jolla, CA) to make the cysteine substitutions of various residues in the M2 and M3 regions of NR1 and NR3 subunits. The in vitro mutagenesis of cDNAs was conducted according to the procedure recommended by the vendor. All point mutations were verified by DNA sequencing of the mutated region. cRNA was transcribed in vitro in the presence of the capping nucleotide using T3 or T7 mMESSAGE mMACHINE transcription kits (Ambion, Austin, TX).

\section{Electrophysiological recordings of recombinant channels from oocytes}

Xenopus oocytes were prepared as described previously (Sullivan et al., 1994). Two-electrode voltage-clamp recordings were performed as described previously at a holding potential of $-60 \mathrm{mV}$ (Chatterton et al., 2002); $10 \mu \mathrm{M}$ glycine was used as the agonist to elicit current from NR1/ NR3A receptors unless otherwise indicated. NR1/NR2/NR3 receptors were not used in this study because of the lack of pharmacological agents to definitively separate them from NR1/NR3 receptors. The extracellular recording solution was composed of Barium Ringer's solution (in mM): 1 $\mathrm{BaCl}_{2}, 95 \mathrm{NaCl}, 2 \mathrm{KCl}, 5 \mathrm{HEPES}, \mathrm{pH}$ adjusted to 7.5 with $\mathrm{NaOH}$. Data were analyzed using PowerLab, version 3.6, and SigmaPlot software (SPSS, Chicago, IL). Solutions and drugs were applied to oocytes with an array of pipettes to achieve rapid solution exchange (complete within 5-8 s). All chemicals were purchased from Sigma (St. Louis, MO) unless otherwise indicated. All experiments were performed in accordance with the policies of the Animal Care and Use Committee at the Burnham Institute for Medical Research.

\section{Chemical modification by methanethiosulfonate agents}

Amino acid residues in the M2 and M3 segments of each subunit were individually mutated to cysteine, and mutant channels were probed with cysteine-specific methanethiosulfonate (MTS) reagents from the extracellular surface of the membrane (Beck et al., 1999). To monitor the size limitation of accessibility, the smaller MTS-ethylammonium (MTSEA) and the larger MTS-ethyltrimethylammonium (MTSET) were used.

Extracellular modification. MTS reagents (MTSEA and MTSET; Toronto Research Chemicals, North York, Ontario, Canada) were used to probe the cysteine-substituted mutant channels from the extracellular side of the membrane. The MTS reagents, with a concentration of 200 $\mathrm{mM}$, were freshly made from powder before each experiment and were kept on ice before any dilution. The ice-cold aliquots of the $200 \mathrm{~mm}$ MTS solution were diluted to the final concentration as indicated immediately before the experiments. Baseline average glycine current amplitude $\left(I_{\text {pre }}\right)$ was obtained by measuring two to three consecutive glycine-activated currents separated by $90 \mathrm{~s}$ washes in glycine-free solution. MTSEA at a concentration of $0.5 \mathrm{~mm}$ was applied in the presence of glycine for $30 \mathrm{~s}$. After MTSEA application, the average amplitude of the glycine-activated current $\left(I_{\text {post }}\right)$ was determined by two to three agonist applications obtained 3 min after modulation to observe a persistent effect. The ratio $\left(I_{\text {post }} / I_{\text {pre }}\right)$, expressed as a percentage, was used to establish the degree of block $\left(1-I_{\text {post }} / I_{\text {pre }}\right)$ or potentiation $\left(I_{\text {post }} / I_{\text {pre }}-1\right) \times 100$. The peak amplitude fluctuated within a range of $10 \%$ of all recordings even with the fast desensitization of the glycine-activated current. Changes $>10 \%$ were analyzed for statistical significance by an unpaired Student $t$ test using the StatView program (SAS Institute, Cary, NC); statistical significance was determined by a value of $p<0.05$. The statistical results from the unpaired Student $t$ test were presented in each histogram. Current amplitudes were corrected for rundown when appropriate.

Kinetic studies. The small size of MTSEA facilitates its accessibility to more cysteine residues, and it is therefore used for our kinetic studies. In parallel experiments, however, using the permanently charged and larger probe, MTSET, the results were similar to those using MTSEA. To accurately measure the reaction rate of the MTSEA modification and to avoid the contamination by the rate of solution exchange, we slowed the on rate of MTSEA reaction by lowering the concentration of MTSEA. The time constant for a complete solution exchange was estimated to be $1-3 \mathrm{~s}$. The concentrations of MTS reagents were therefore selected to achieve a time constant of at least $10 \mathrm{~s}$. Two protocols were used: (1) the continuous protocol for the glycine-activated current with minimal desensitization after mutation, or (2) the pulsive protocol for the fast desensitizing glycine-activated current. Reaction rates, measured at $-60 \mathrm{mV}$, were determined either by measuring the rate of change in current amplitudes during a continuous application of the MTSEA reagent ("continuous" protocol) (see Fig. $5 A$ ), or by applying a reagent for a specified amount of time and measuring current amplitudes before and after this application ("pulsive" protocol) (see Fig. 5B) (Sobolevsky et al., 2002a). For both protocols, changes in current amplitudes were fitted with a single exponential by SigmaPlot software using the Marquardt-Levenberg algorithm to achieve the best fit. The equation used for fitting the on-rate of MTSEA modification was as follows:

$$
I=I_{\infty}+\left(I_{0}-I_{\infty}\right) \times \exp (-t / \tau)
$$

where $t$ is the cumulative time of exposure to the reagent, $I$ is the peak current after $t$ seconds of this exposure, $I_{0}$ is the initial current amplitude, $I_{\infty}$ is the asymptotic current amplitude when the reaction is complete, and $\tau$ is the time constant. The apparent second-order rate constant for chemical modification, $k$, was calculated by the following:

$$
k=1 /(\tau \times[\mathrm{MTS}]),
$$

where [MTS] is the concentration of the MTS reagent. Current amplitudes were corrected for rundown when appropriate.

For the kinetic study of NR1/NR3A mutant channels in the closed state, traditional glycine site antagonists (e.g., 5,7-dichlorokynurenic acid and 2-carboxyindole analogs) could not be used to ensure that the channel stayed in a closed state. They did not completely block glycineevoked currents at $<10 \mu \mathrm{M}$ and, in fact, activated small currents as partial agonists at concentrations $>10 \mu \mathrm{M}$ (H.-S. V. Chen and H. Takahashi, unpublished data). Because there are no known full competitive antagonists for NR1/NR3A channels, the modification rate of MTS reagents in the closed (unliganded) conformation was assessed in the absence of glycine. For the kinetic analysis in Figure 5, most of mutant channels displayed baseline currents close to zero, indicating that they were mostly in the closed state; thus, the addition of antagonist would not have closed the channels further, alleviating the need for a full competitive antagonist in these experiments. For evaluating the reaction rates in the closed and open conformations using a pulsive protocol, the durations of the application of the MTS reagent were progressively prolonged to ensure that a steady state was obtained for curve-fitting purposes (usually three to four applications of $10-15 \mathrm{~s}$ each, then three to four applications of $20 \mathrm{~s}$ each, and followed by $30-60 \mathrm{~s}$ applications as needed). To study the open state with pulsive protocols, MTSEA and glycine were applied together as in the bottom tracing of Figure $3 B$. The protocol by Sobolevsky et al. 


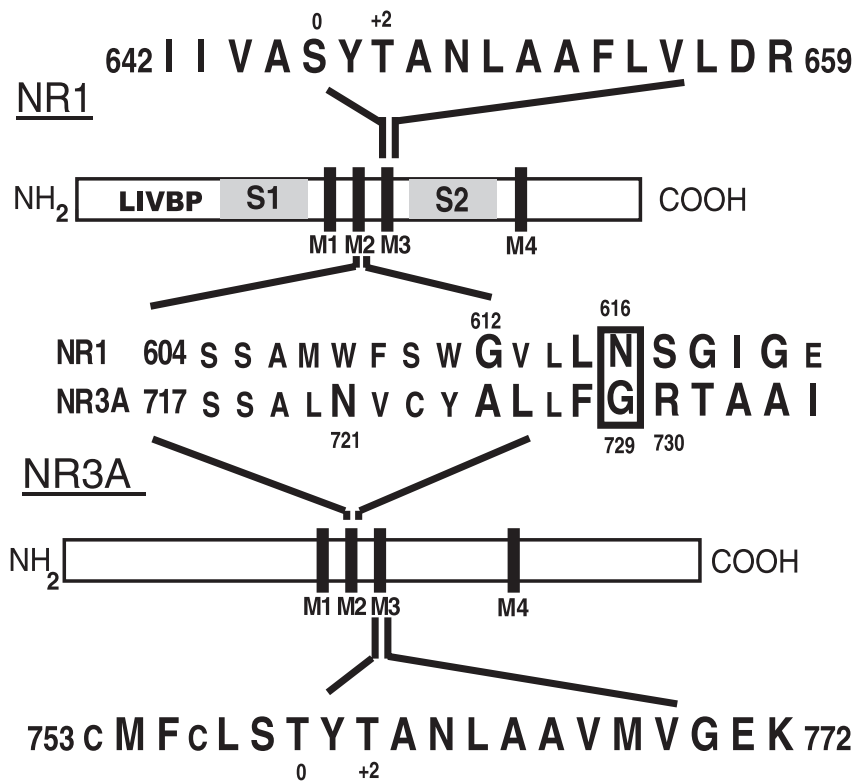

Figure 1. Diagram of cysteine substitutions in the NR1 and NR3A subunits. Schematic representation of the NR1 and NR3A subunits indicating the locations of amino acid residues that were substituted with cysteines in the experiments. Residues substituted with cysteines are shown in larger fonts. The N-site residues in the NR1 subunit (N616) and the NR3A subunit (G729) are highlighted. S1 and S2 represent the agonist binding domains. LIVBP (leucineisoleucine-valine binding protein) is the N-terminal modulatory domain that has homology to bacterial amino acid binding proteins.

(2002b), to evaluate the rate of open-state modification by applying MTSEA in the middle of agonist application, was also used and did not change the result. This latter protocol was deemed unnecessary because of the minimal and quickly reversible open-channel block by the MTS reagents at concentrations of $\leq 250 \mu \mathrm{M}$.

Persistent zinc coordination

We initially tested $\mathrm{Cd}^{2+}, \mathrm{Cu}^{2+}, \mathrm{Ni}^{2+}$, and $\mathrm{Zn}^{2+}$, at concentration of 0.25-0.5 mM, for multivalent coordination of vicinal cysteine residues. $\mathrm{Cd}^{2+}$ and $\mathrm{Ni}^{2+}$ blocked the wild-type and all mutant channels only slightly with similar magnitude and rapid reversible kinetics. At concentrations $>1 \mu \mathrm{M}, \mathrm{Cu}^{2+}$ produced persistent inhibition in the wild-type NR1/NR3A channels. Zinc (at 100 and $250 \mu \mathrm{M}$ ) reversibly blocked NR1/ NR3A glycine-gated channels ( $>90 \%$ ), and the current returned to baseline $( \pm 5 \%)$ within $30 \mathrm{~s}$ after zinc washout. Zinc was the only ion tested that showed persistent coordination with vicinal cysteines in NR1/NR3A mutant channels. NR1(T648C) and NR3A(T761C) are the homologous substitution at the +2 position of the M3 segments. To minimize the influence of desensitization of NR1/NR3A receptors, the current amplitude after persistent block by zinc coordination was measured $30 \mathrm{~s}$ after zinc washout $\left(I_{\text {post }}\right)$, and compared with the control current at the same time point $\left(I_{\text {pre }}\right)$. The persistent degree of block by zinc was calculated as follows: $\left(1-I_{\text {post }} / I_{\text {pre }}\right) \times 100 \%$. The commonly used zinc chelating agent, tricine (Paoletti et al., 1997), produced nonspecific blockade of these NR1/NR3A mutant channels and therefore could not be used to reverse the persistent blockade of zinc.

\section{Results}

We performed cysteine substitutions on amino acid residues in the M2 and M3 segments of NR1-1a and NR3A subunits (Fig. 1 illustrates the relative positions of residues selected for cysteine substitution). We then coexpressed these subunits in Xenopus oocytes to form glycine-activated excitatory channels (Chatterton et al., 2002). Amino acid residues in the M2 and M3 segments of each subunit were individually mutated to cysteine, and mutant channels were probed with cysteine-specific methanethio-
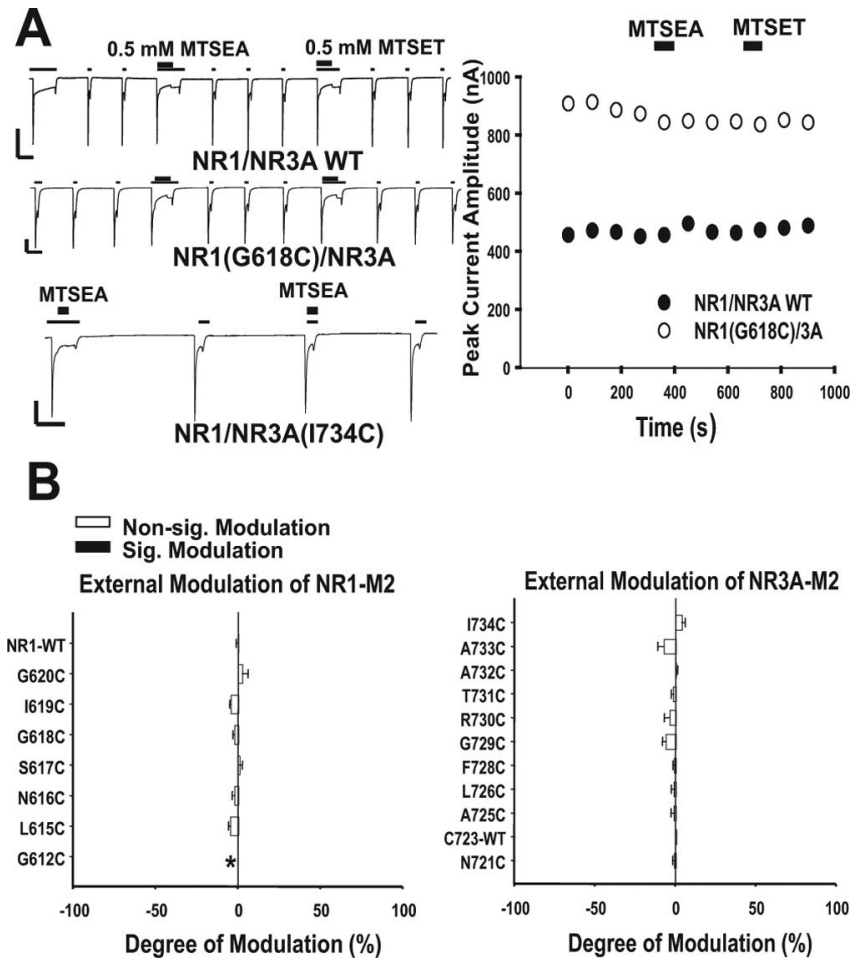

Figure 2. Lack of persistent modulation by external MTSEA of cysteine-substituted mutations in the M2 segment of NR1/NR3 channels. $A$, Extracellular MTSEA ( $0.5 \mathrm{~mm}$ applied for $30 \mathrm{~s}$ ) did not persistently affect wild-type or M2-segment mutant NR1/NR3A channels activated by $10 \mu \mathrm{m}$ glycine (thin line). Left, Representative recordings of the wild-type and NR1(G618C)/ NR3A mutant channels are shown (top and middle traces). If MTSEA did not produce a persistent effect, $0.5 \mathrm{~mm}$ MTSET was also applied for $30 \mathrm{~s}$ but was also found to have no effect. Repetitive application of MTSEA to NR1/NR3A(1734C) (bottom trace) or simultaneous application of glycine and MTSEA (third glycine-elicited current trace) do not affect this result. Calibration: 200 $\mathrm{nA}, 30$ s. Right, Peak current amplitude at $-60 \mathrm{mV}$ plotted against time. $\boldsymbol{B}$, Summary of percentage change in peak current amplitude after exposure to MTSEA. Degree of block or potentiation (\%) (as defined in the Materials and Methods) was used to illustrate the degree of modulation by MTS reagents. Each bar represents mean \pm SEM from 3-10 experiments with persistent inhibition to the left and potentiation to the right. Mutant channels not significantly modified by MTSEA or not statistically different from the wild type are shown as open bars (nonsig. modulation). NR1(N616) and NR3A(G729) are N-site equivalents. Experiments on NR1(G612C)/NR3A (asterisk) could not be reliably performed because of small glycine-activated currents $(<50 \mathrm{nA})$.

sulfonate reagents from the extracellular surface of the membrane (Beck et al., 1999).

\section{Cysteine substitution mutants of the M2 segment form} functional NR1/NR3A channels

NR1(N616) and NR3A(G729) represent the N-site equivalents in the M2 region. Ten residues surrounding NR3A(G729) and seven residues around NR1(N616) in the M2 segment of each subunit were chosen and individually mutated to cysteine for MTSEA modification based on the published accessibility pattern of NR1/NR2C channels (Kuner et al., 1996). The selected amino acid residues in the NR1 or NR3A subunits represent the presumed tip of the reentrant pore loop, and thus are potentially accessible to external MTS reagents. Additionally, NR3A possesses several intrinsic cysteines in the M2 and M3 segments: one in M2 and two in M3, whose function was also assessed. When coexpressed with the NR1 subunit, all 10 NR3A mutants, with the exception of F728, produced glycine-activated currents comparable with wild-type NR1/NR3A channels with regard to the amplitude and gating kinetics (Fig. 2). Mutant channels containing 
NR3A(F728C) manifested a larger tail current, suggesting a change in gating kinetics.

When coexpressed with the NR3A subunit, all NR1 mutants in the M2 segment yielded glycine-activated currents with current characteristics and kinetics comparable with those of wild type. Except for channels containing NR1(G618C), peak current amplitudes were slightly smaller than those of wild-type NR1/ NR3A channels. Because peak currents observed after NR1 (G618C) mutation were much smaller $(<50 \mathrm{nA})$, they were not analyzed further for modification by MTS agents.

\section{Effect of extracellular MTS reagents on cysteine-substituted M2 segments of NR1/NR3A channels}

Initially, we investigated the accessibility to MTS reagents of residues in the M2 segments of NR1/NR3A channels. In wild-type NR1/NR3A channels, there was no persistent effect of extracellular MTS reagents in the open or closed state (Fig. 2A). Strikingly, none of the channels bearing mutated M2 segments in the NR1 or NR3A subunit could be modified by externally applied MTSEA or MTSET. The concentration of MTS reagents $(<1 \mathrm{mM})$ was chosen to avoid nonspecific channel block or membrane destabilization (baseline shift) in the oocyte expression system. The duration of MTS reagent application was set at $30 \mathrm{~s}$ to limit potential transmembrane effects because MTSEA is membrane permeable. The published reaction rate of exposed cysteine residues with MTSEA is $10^{2}-10^{5} \mathrm{M}^{-1} \mathrm{~s}^{-1}$ (Liu et al., 1997; Sobolevsky et al., 2002a). Hence, this duration of exposure to MTSEA should have been sufficient to allow significant reaction with water accessible cysteine residues $(>1.5$ times the reaction time constant). Because of the fast desensitization of NR1/NR3A currents, a $10-15 \mathrm{~s}$ exposure to $10 \mu \mathrm{M}$ glycine was used to elicit maximal peak currents, followed by a $90 \mathrm{~s}$ washout period between applications to ensure complete recovery. The current amplitude was calculated from the mean of two to three applications of glycine before and after the MTS modification. There was no observed modification by either MTSEA or MTSET of any of the cysteinesubstituted mutants of the M2 segment for the NR1 and NR3 subunits (Fig. 2A). Application of MTSEA and glycine simultaneously to elicit the current did not affect this result, indicating the lack of reaction with MTSEA was not attributable to the fast desensitization of the NR1/3A current (Fig. 2A, bottom trace). Additional exposure to external MTSEA after the first application of an MTS reagent did not alter this result (Fig. $2 \mathrm{~A}$, bottom trace). All of these procedures should have maximized our ability to see a modification by MTS reagents of accessible cysteine residues. Yet, we observed no modifications by either MTSEA or MTSET of any of the cysteine-substituted mutants in the M2 segment for either the NR1 or NR3 subunits. Figure $2 B$ summarizes these results. Because of the lack of effect of external MTS reagents on the presumed tip of the pore loop, extensive consecutive mutations of the M2 segment were not undertaken. Instead, we turned to the M3 segment to determine whether its structure might be somehow affecting accessibility of MTS reagents to the pore loop within the deeper M2 region of the channel. Indeed, this was found to be the case, as shown below.

\section{Cysteine-substituted mutants of the M3 segment form functional NR1/NR3A channels}

Among the various domains found in glutamate receptors, the M3 region contains the most conserved motif, SYTANLAAF, which has been suggested to function as a transduction element for channel gating (Jones et al., 2002). The serine residue in this motif is designated as position $0(\mathrm{~S}+0)$ to simplify the description of the mutated residues (Fig. 3 ). In the NR3A subunit, the $\mathrm{S}+0$ residue of this motif is a threonine, and a valine replaces the phenylalanine $(\mathrm{F})$ at the +8 position. We substituted cysteine for a stretch of 18 consecutive residues in the M3 segment of either the NR1 or NR3A subunit. NR3A also contains two endogenous cysteine residues in the M3 segment; the results of MTS modification of a total of 20 aa are therefore presented in this study.

When coexpressed with wild-type NR1, all of the mutations in the M3 segment of the NR3A subunit, except NR3A $(A+3 C)$ and $\mathrm{NR} 3 \mathrm{~A}(\mathrm{~L}+5 \mathrm{C})$, produced glycine-evoked inward currents at a holding potential of $-60 \mathrm{mV}$. Glycine-induced currents from wild-type NR1/NR3A receptors manifested a fast initial transient (rapid decay after the peak current) and a small tail current after washout of glycine (Fig. $3 \mathrm{~A}$, bottom trace at right). Some cysteine substitution mutations in the M3 segment of the NR3A subunit, however, modified the characteristics of the current in one of three ways: (1) similar fast transients but larger tail currents [L-2C, T+2C, and G + 11C mutations, indicated by diamonds in Figure $3 A$ and shown in the current trace from NR1/NR3A(L2C) channels]; (2) minimal fast transients and smaller or no tail currents, suggesting lack of desensitization $[\mathrm{Y}+1 \mathrm{C}, \mathrm{N}+4 \mathrm{C}$, $\mathrm{A}+6 \mathrm{C}, \mathrm{A}+7 \mathrm{C}, \mathrm{M}+9 \mathrm{C}, \mathrm{V}+10 \mathrm{C}$, and $\mathrm{K}+13 \mathrm{C}$ mutations, indicated by gray circles and illustrated in the current trace of NR1/ NR3A(N+4C) channels]; or (3) large leak currents, which could be blocked by replacing extracellular $\mathrm{Na}^{+}$with the large impermeant molecule $N$-methyl-D-glucamine (NMDG) (96 mM), suggesting a constitutively open channel $(\mathrm{A}+3 \mathrm{C}$ and $\mathrm{L}+5 \mathrm{C}$ mutations). These constitutive leak currents also decreased $60-70 \%$ after application of $10 \mu \mathrm{M}$ glycine and manifested slow recovery after agonist washout [Fig. 3A, currents from NR1/NR3A(L+5C) channels shown at top right]. Compared with wild-type NR1/ NR3A currents, NR3A mutations $\mathrm{N}+4 \mathrm{C}, \mathrm{A}+6 \mathrm{C}$, and $\mathrm{G}+11 \mathrm{C}$ manifested currents of smaller amplitude (Fig. 3A, asterisks). These findings suggest the possibility that NR3A plays a modulatory role (see below). Other mutations resulted in minimal changes in current shape or amplitude. A systematic analysis of the effects of cysteine substitutions on the M3 segment of the NR1 and NR3A subunits is shown in Figure $3 A$.

For mutations in the M3 segment of the NR1 subunit, any mutation internal to the $\mathrm{S}+0$ position yielded currents comparable in shape and amplitude to the wild type. The $\mathrm{T}+2 \mathrm{C}, \mathrm{A}+3 \mathrm{C}$, and $\mathrm{L}+11 \mathrm{C}$ mutations resulted in larger tail currents ["type 1" changes described above; NR1(T+2C)/NR3A current is shown in Fig. $3 A$, top left]. The $\mathrm{S}+0 \mathrm{C}, \mathrm{Y}+1 \mathrm{C}, \mathrm{N}+4 \mathrm{C}, \mathrm{A}+6 \mathrm{C}, \mathrm{L}+9 \mathrm{C}$, and $\mathrm{V}+10 \mathrm{C}$ mutations decreased or eliminated the fast decay ["type 2" changes; NR1(S+0C)/NR3A current is shown in Fig. $3 A$, lower left]. The $\mathrm{N}+4 \mathrm{C}$ and $\mathrm{A}+6 \mathrm{C}$ mutations yielded currents of smaller amplitude compared with the glycine-evoked currents of wild-type NR1/NR3 receptors. Other mutations in the M3 region of the NR1 subunit did not create significant changes. All NR1 and NR3A M3 segment mutant channels manifested magnesium insensitivity comparable with that of wild-type NR1/ NR3A channels. Although there were some macroscopic changes in the shape of the currents evoked from these cysteinesubstituted mutant channels, most of the properties and therefore the inferred structure of the channels appeared to remain similar to that of wild type based on the comparable amplitude of glycine-activated currents and relative insensitivity to magnesium blockade. Next, we focused on the accessibility pattern of the entire M3 segment determined by the SCAM. A cysteine mutant was considered to be exposed to the water-filled lumen if there was a persistent effect on the peak or sustained current amplitude after the application of an MTS reagent. 


\section{Effect of extracellular MTS reagents on the M3 segment of cysteine-substituted NR1/NR3A channels}

As mentioned above, there was no persistent effect of extracellular MTS reagents on wild-type NR1/NR3A channels. MTS reagents, at concentrations of $\leq 0.5 \mathrm{~mm}$ in the presence of glycine, produced only minimal and quickly reversible openchannel block (Fig. 3B, middle trace). Representative tracings of potentiation or inhibition by MTSEA are shown in Figure $3 B$ (top and middle traces). Note, however, that MTSEA had dual effects on the NR1/ NR3A $(T+2 C)$ mutant, initially decreasing glycine-activated peak current amplitude, but subsequently potentiating the current (Fig. 3B, bottom trace). Figure 4 summarizes the results of accessibility experiments on the M3 segment. In the presence of glycine, the $\mathrm{T}+2$ position of either subunit, represented by NR1(T648C) or NR3A(T761C), is located at the deepest position within the channel vestibule that was accessible to MTS modification. Cysteine substitutions of amino acids more internal than the $\mathrm{T}+2$ position were not affected by MTS reagents.

The effect of MTSEA modification on cysteine-substituted residues in the M3 segments of the NR1 and NR3A subunits, whether categorized by inhibition or potentiation, was similar at each location except at the $\mathrm{T}+2$ and $\mathrm{L}+5$ positions (Fig. 4). MTSEA modification at these two positions in NR1 blocked glycine-evoked currents. MTSEA modification at the $\mathrm{NR} 3 \mathrm{~A}(\mathrm{~T}+2 \mathrm{C})$ position initially decreased but subsequently potentiated the current amplitude (as described above). In contrast, in the case of the NR3A (L+5C) mutant, external MTSEA manifested fast potentiation followed by slow block (initial potentiation shown in Fig. 4). Overall, in our experiments, the effects of MTSEA modification on residues external to the +5 position in the M3 segment of either NR1 or NR3A was similar to that previously reported for the NR1 subunit in NR1/NR2 receptors (Beck et al., 1999; Sobolevsky et al., 2002b). This result suggests that the M3 segments of both NR1 and NR3 subunits in NR1/NR3A receptors have a configuration similar to that of the NR1 subunit in NR1/NR2 receptors. In contrast, compared with NR1/NR2 channels, the different modification pattern of MTSEA on residues internal to the +5 position in NR1/NR3A channels suggests the presence of a distinct structural feature in this region. Further along these lines, compared with MTSEA, MTSET manifest a more limited accessibility pattern to the M3 segment of the NR1 subunit of NR1/NR3 receptors (but not NR1/NR2 receptors), suggesting a narrowed outer vestibule of the channel or possibly a modified microenvironment of the outer vestibule (see Discussion).

Of note, MTSEA modulation of the $+3,+4,+6,+10$, or +12
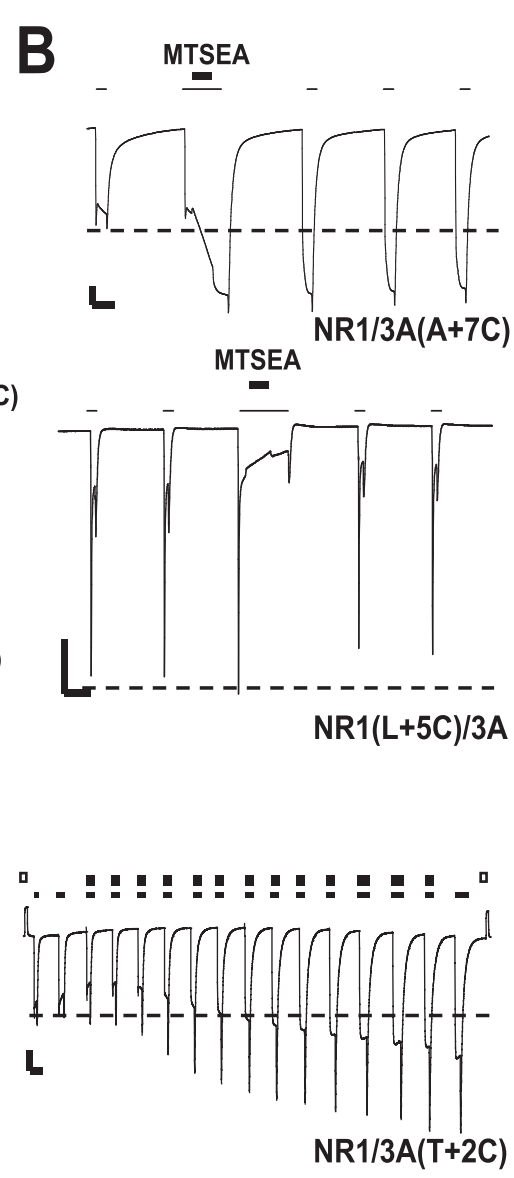

NR1/3A WT
$N R 1 / 3 A(T+2 C)$

Figure 3. MTS modulation of glycine-activated currents from cysteine-substituted mutations of NR1/NR3A M3 segments. $\boldsymbol{A}$, Representative recordings of glycine-elicited currents from channels containing cysteine substitutions in the M 3 segments. The open circles indicate mutant channels that had minimal changes in current shape compared with the wild-type NR1/NR3A channels (NR1/NR3A WT). The diamonds indicate mutant channels with larger tail currents than wild type [e.g., NR1/NR3A(L-2C)

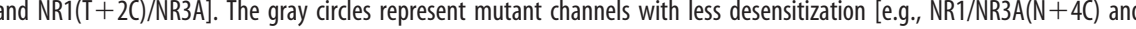
$\mathrm{NR} 1(\mathrm{~S}+\mathrm{OC}) / \mathrm{NR} 3 \mathrm{~A}]$. The squares represent mutant channels exhibiting large baseline leak current (because of constitutively open M3 segment mutations. MTSEA ( $0.5 \mathrm{~mm}$ ) (thick line) was applied for $30 \mathrm{~s}$ in the presence of $10 \mu \mathrm{m}$ glycine (thin line) to allow

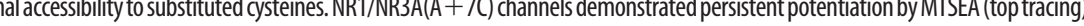
(dashed lines). A pulsive protocol with repetitive coapplication of $10 \mu \mathrm{m}$ glycine and $250 \mu \mathrm{m}$ MTSEA (see Materials and Methods) revealed dual effects of MTSEA on NR1/NR3A(T $+2 C$ ) channels (bottom tracing). NMDG-substituted external solution (96 mM NMDG

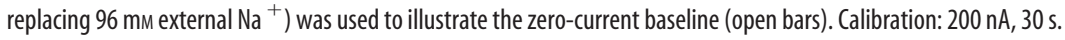

positions of either subunit (Fig. 4) or of the NR1(A+7C) position resulted not only in potentiation or inhibition of peak glycineevoked currents but also in an increase in the baseline leak current. This increase in leak current suggests either a change in gating (Jones et al., 2002) or the presence of a channel locked in a partially open conformation (Chen et al., 2005). Elucidation of the mechanism of this additional effect of MTSEA modulation will require additional investigation of the gating mechanism and was not further pursued here.

State-dependent changes in the modification rate of cysteine-substituted M3 segments of NR1/NR3A channels during gating

We next examined possible molecular rearrangements of the outer vestibule of the NR1/NR3 channel by comparing the on- 
A Non-sig. Modulation
Sig. Modulation

External MTSEA Modulation of NR1-M3 External MTSET Modulation of NR1-M3
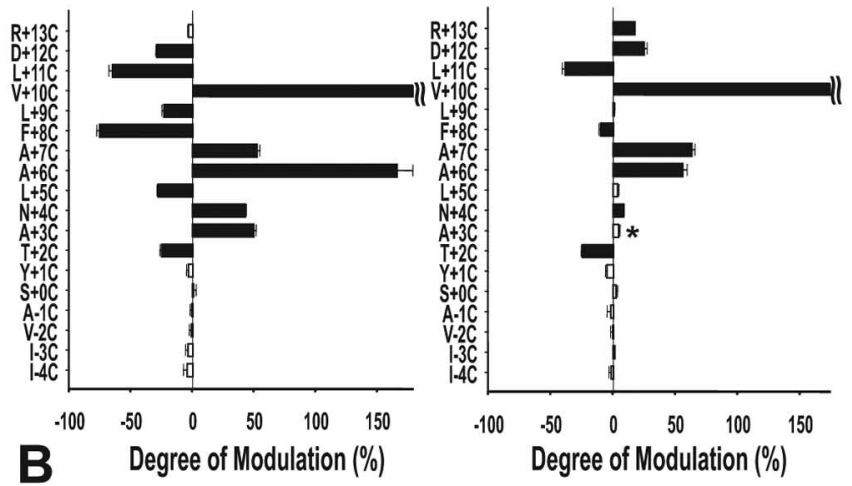

External MTSEA Modulation of NR3A-M3 External MTSET Modulation of NR3A-M3
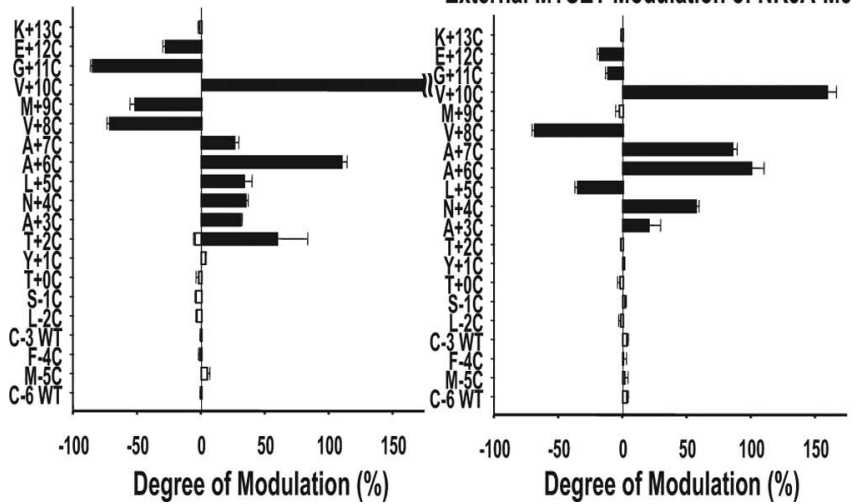

Figure 4. Accessibility pattern of cysteine-substituted mutations in the M3 segments of NR1 and NR3 subunits. Summary of percentage change in current amplitude after exposure to MTS reagents $(0.5 \mathrm{~mm}$ for $30 \mathrm{~s})$. Each bar represents the mean \pm SEM from 3-12 experiments with persistent inhibition to the left and potentiation to the right. Compared with wild-type NR1/ NR3 channels, statistically significant changes of mutant channels are presented as filled bars (sig. modulation; $p<0.05$ ). Mutant channels not significantly modified by or not statistically different from wild-type are shown as open bars (nonsig. modulation). MTSEA slightly decreased the peak amplitude current of NR1(T+2C)/NR3A (open bar pointing left) but significantly increased the sustained component of current (filled bar pointing right). NR1(S646) and NR3A(T759) represent equivalent residues at the +0 position of the conserved SYTANLAAF motif. The $A+3$ residue in the NR1 subunit was the only residue found to react with $0.5 \mathrm{~mm}$ MTSET slowly but underwent significant modification with prolonged application (asterisk).

rate of MTSEA modification in the presence and absence of glycine, as described for classical NMDARs (Sobolevsky et al., 2002a). Continuous and pulsive protocols (see Materials and Methods) were applied to measure the on-rate of MTSEA modification (Fig. $5 A, B$ ). The concentration of MTSEA was adjusted to slow down the on-rate to reduce possible confounding errors arising from the rate of solution exchange in the oocyte recording system. Because of the lack of a complete antagonist for the glycine-activated current, the on-rate of MTSEA modification of the closed state of the channel was assessed in the absence of glycine but without a competitive antagonist (see Materials and Methods). The peak current (or sustained current if there was no fast transient current) amplitudes of glycine-activated responses were plotted against the cumulative time of MTS exposure, providing the time course of MTS modification. Single exponential fit for the time course of MTS modification was then performed to obtain the rate constants of reactions (Fig. $5 B$, bottom graph). The $\mathrm{T}+2, \mathrm{~A}+3, \mathrm{~A}+7$, and $\mathrm{V}+10$ mutations of the NR1 subunit
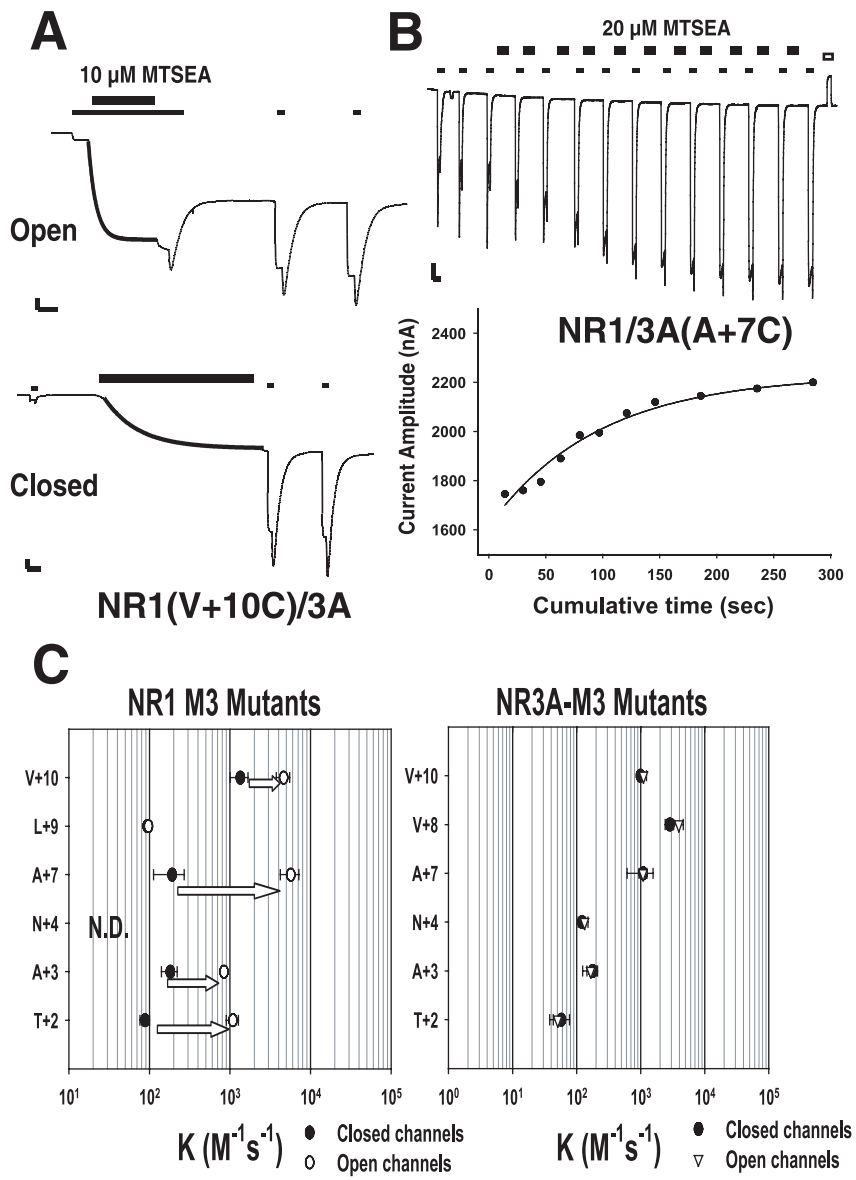

Figure 5. Modification rates by MTSEA of cysteine-substituted mutations in the M 3 segment in the presence versus the absence of glycine. $A$, Reaction of MTSEA with the mutant channel NR1 $(V+10 C) / N R 3 A$. The reaction curve for potentiation could be fit by a single exponential. The resulting time constant was used to calculate the apparent rate constant (summarized in C). MTSEA modulation was tested with a continuous application protocol (see Materials and Methods). MTSEA (10 $\mu \mathrm{m})$ was applied continuously in the presence or absence of $10 \mu \mathrm{m}$ glycine. Because the NR1 $(V+10 C)$ residue is located at a very external location (the +10 position), this residue was easily accessible to external MTSEA in the presence or absence of agonist. The $\mathrm{NR} 1(\mathrm{~V}+10 \mathrm{C})$ residue was therefore chosen to illustrate the significant difference in reaction rates between the open and closed states. Calibration: $200 \mathrm{nA}, 30$ s. B, Pulsive protocol for MTSEA modulation. To measure the modification rate of MTSEA in the absence of glycine, the MTSEA was applied for $10-60 \mathrm{~s}$ (thick bars) between consecutive glycine-elicited currents (thin bars) until minimal modification by MTSEA was observed. The peak current amplitude for each glycine-activated current was then plotted against the cumulative time of exposure to MTSEA. The data points were then fitted by a single exponential function, and the apparent rate constant was calculated (see Materials and Methods). C, Comparison of second-order modification rate constants by MTSEA of various mutant channels in the M3 segment. Each data point represents three to six experiments and is presented as mean \pm SEM. Reaction of MTS reagents at the $\mathrm{N}+4$ position in the NR1 subunit could not be evaluated because it never reached a steadystate value. The arrows indicate a significant change $(p \leq 0.05)$ in rate constants between the presence (open state) and absence of glycine (closed state). There were no significant statedependent changes in the rate constants for any of the NR3 M3 mutants tested.

manifested faster reaction rates with MTS reagents in the presence versus the absence of glycine (Fig. 5C, left); this result was predictable based on the molecular rearrangements that occur during the gating process, as shown previously for NR1/NR2 receptors (Sobolevsky et al., 2002a). This result is also consistent with the notion that this region constricts when the channel is in the closed conformation.

Surprisingly, there was no state-dependent change in modification rates for the M3 mutants of the NR3A subunit in the 
presence of glycine (Fig. $5 C$, right). The absolute estimated reaction rates, however, became faster for residues closer to the extracellular surface. This result cannot be explained by lipid accessibility of membrane-permeable MTSEA because permanently charged MTSET also modified these residues in the M3 segment (Fig. 4). Moreover, we found that charged zinc ions could coordinate $\mathrm{T}+2$ cysteine-substituted residues (see below), suggesting a surface rather than a submembrane location of these residues. As discussed below, these results suggest that the role of the NR3 subunit in NR1/NR3A receptors is to modulate channel properties rather than to gate transduction in response to agonist binding at micromolar concentrations.

\section{Zinc coordination by substituted cysteines occupying homologous positions in NR1 and NR3A subunits} Cysteine residues have the ability to coordinate multivalent cations in proteins (Holm et al., 1996). To rule out a lipid-accessible effect of membrane-permeable MTSEA and further elucidate the structure of the outer vestibule of the channel, we used permanently charged cations to coordinate the substituted cysteines. Previous coordination experiments of substituted cysteines by $\mathrm{Cu}^{2+}$ in NR1 and NR2C subunits had determined that the M3 regions of these two subunits were staggered by $\sim 4$ aa (Sobolevsky el al., 2002b). We hypothesized that the deepest positions exposed to MTS reagents, located at NR1 $(\mathrm{T}+2 \mathrm{C})$ and $\mathrm{NR} 3 \mathrm{~A}(\mathrm{~T}+2 \mathrm{C})$, represented the narrowest portion of the external vestibule or mouth of the channel; thus, these positions should have the requisite proximity to coordinate a divalent cation. A single mutation of each subunit, coexpressed with the corresponding wild-type subunit, was initially tested, and then double mutant channels formed by $\mathrm{NR} 1(\mathrm{~T}+2 \mathrm{C}) / \mathrm{NR} 3 \mathrm{~A}(\mathrm{~T}+2 \mathrm{C})$ were tested to determine whether a divalent cation could coordinate the neighboring cysteines. Zinc was the only divalent cation that we found could produce a persistent modification of glycineactivated currents mediated by $\mathrm{NR} 1(\mathrm{~T}+2 \mathrm{C}) / \mathrm{NR} 3 \mathrm{~A}(\mathrm{~T}+2 \mathrm{C})$ channels.

In wild-type NR1/NR3A channels, zinc rapidly inhibited glycine-activated currents when applied in the presence of agonist; this effect was quickly and completely reversible after washout. There was no persistent effect of zinc coordination on wildtype NR1/NR3A channels. Additionally, the single $\mathrm{T}+2 \mathrm{C}$ substitution in either the NR1 or NR3A subunit yielded little persistent inhibition of the current measured $30 \mathrm{~s}$ after $\mathrm{Zn}^{2+}$ washout. A similar reversible effect of $\mathrm{Zn}^{2+}$ was observed on NR1/NR3A channels and on channels containing single $\mathrm{T}+2 \mathrm{C}$ substitution in the NR1 or NR3A subunit; therefore, only one representative tracing is shown in Figure $6 \mathrm{~A}$ (left top panel). In contrast to the rapidly reversible inhibition of wild-type and single $\mathrm{T}+2 \mathrm{C}$ substituted channels, zinc produced persistent (or slowly reversible) inhibition of glycine-activated currents from $\mathrm{NR} 1(\mathrm{~T}+2 \mathrm{C}) / \mathrm{NR} 3 \mathrm{~A}(\mathrm{~T}+2 \mathrm{C})$ channels, and this inhibition did not completely recover after $\mathrm{Zn}^{2+}$ washout. The degree of persistent block by zinc for NR1(T+2C)/NR3A, NR1/ $\mathrm{NR} 3 \mathrm{~A}(\mathrm{~T}+2 \mathrm{C})$, and $\mathrm{NR} 1(\mathrm{~T}+2 \mathrm{C}) / \mathrm{NR} 3 \mathrm{~A}(\mathrm{~T}+2 \mathrm{C})$ channels was $4.5 \pm 1.1,14.7 \pm 1.1$, and $37.4 \pm 2.2 \%$, respectively. However, after pretreatment with $0.5 \mathrm{~mm}$ MTSEA for $1 \mathrm{~min}$ to covalently modify the substituted cysteine residues, persistent block by zinc of NR1 $(\mathrm{T}+2 \mathrm{C}) / \mathrm{NR} 3 \mathrm{~A}(\mathrm{~T}+2 \mathrm{C})$ channels decreased to $5.4 \pm 1.5 \%$ (Fig. 6B). We conclude therefore that the residue at the $\mathrm{T}+2$ position was exposed to a water-accessible surface in either the NR1 or NR3A subunit. Of note, zinc did not manifest persistent inhibition of $\mathrm{NR} 1(\mathrm{~A}+3 \mathrm{C}) / \mathrm{NR} 3 \mathrm{~A}(\mathrm{~T}+2 \mathrm{C})$ double mutant channels (data not shown). The $\mathrm{NR} 1(\mathrm{~A}+3 \mathrm{C})$ residue is misaligned
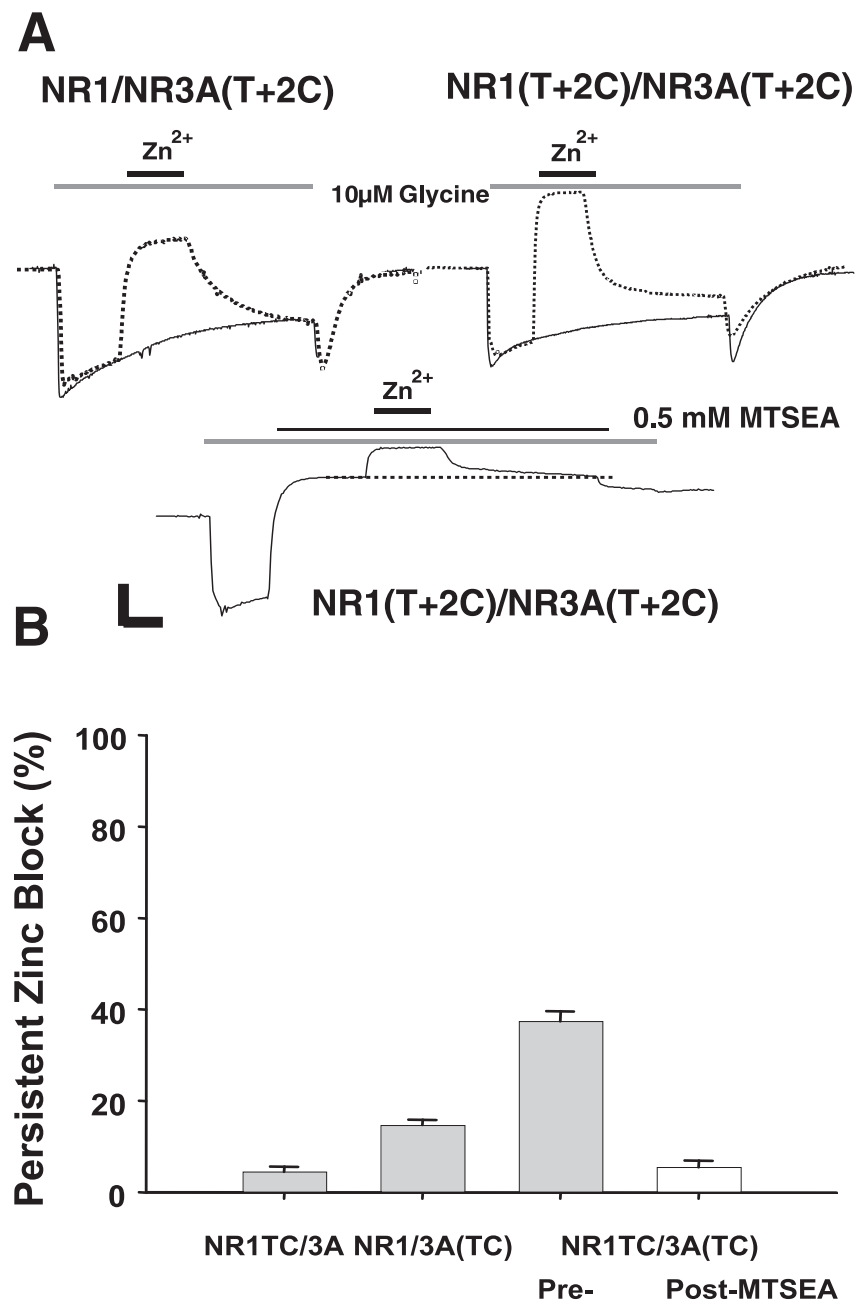

NR1/NR3A Mutants

Figure 6. Persistent zinc inhibition after dual cysteine substitutions at the homologous +2 position in the M3 segments of NR1 and NR3A subunits. $\boldsymbol{A}$, Representative tracings of zinc inhibition/coordination of mutant NR1/NR3A channels. NR1(TC) represents NR1 $(T+2 C)$, and $N R 3 A(T C)$ represents the homologous substitution, $N R 3 A(T+2 C)$. Cysteine substitution at these threonine positions resulted in a leak component of current, which could be reversibly blocked by $250 \mu \mathrm{m} \mathrm{Zn}{ }^{2+}$ or NMDG in the external solution. Additionally, zinc (thick line) reversibly blocked glycine-activated currents from NR1/NR3A $(T+2 C$ ) (top left) and wild-type channels; the current returned to baseline within $30 \mathrm{~s}$ after zinc washout. $\mathrm{Zn}^{2+}$ also produced a persistent (or slowly reversible) component of inhibition of currents from NR1(T+2C)/ $\mathrm{NR3A}(\mathrm{T}+2 \mathrm{C})$ channels (top right). Preapplication of $0.5 \mathrm{~mm}$ MTSEA resulted in persistent inhibition of current from NR1 $(T+2 C) / N R 3 A(T+2 C)$ channels and also prevented persistent coordination of $\mathrm{Zn}^{2+}$ (bottom trace). Calibration: $500 \mathrm{nA}, 30$ s. B, Summary of the degree of persistent blockade by zinc of NR1 $(T+2 C) / N R 3 A$, NR1/NR3A(T+2C), and NR1(T+2C)/ $\mathrm{NR3A}(\mathrm{T}+2 \mathrm{C})$ channels. NR1/NR3A(T+2C) channels manifested a small degree of persistent inhibition by $250 \mu \mathrm{M} \mathrm{Zn}{ }^{2+}$, but persistent inhibition was greatly enhanced by concomitant $\mathrm{NR} 1(\mathrm{~T}+2 \mathrm{C})$ substitution; $100 \mu \mathrm{m} \mathrm{Zn}^{2+}$ produced similar results (data not shown). The persistent block by zinc of NR1 $(T+2 \mathrm{C}) / \mathrm{NR} 3 \mathrm{~A}(\mathrm{~T}+2 \mathrm{C})$ channels was mostly eliminated by pretreatment with $0.5 \mathrm{~mm}$ MTSEA for $1 \mathrm{~min}$. Each column represents mean \pm SEM for three to four experiments.

with the NR3A $(T+2 \mathrm{C})$ residue by only 1 aa. Together, these results for zinc coordination suggest, in contrast to NR1/NR2 channels, that the M3 segments of the NR1 and NR3A subunits of NR1/NR3 channels are aligned symmetrically, with the $\mathrm{NR} 1(\mathrm{~T}+2)$ and $\mathrm{NR} 3 \mathrm{~A}(\mathrm{~T}+2)$ positions forming the narrowest portion of outer vestibule of the channel. 


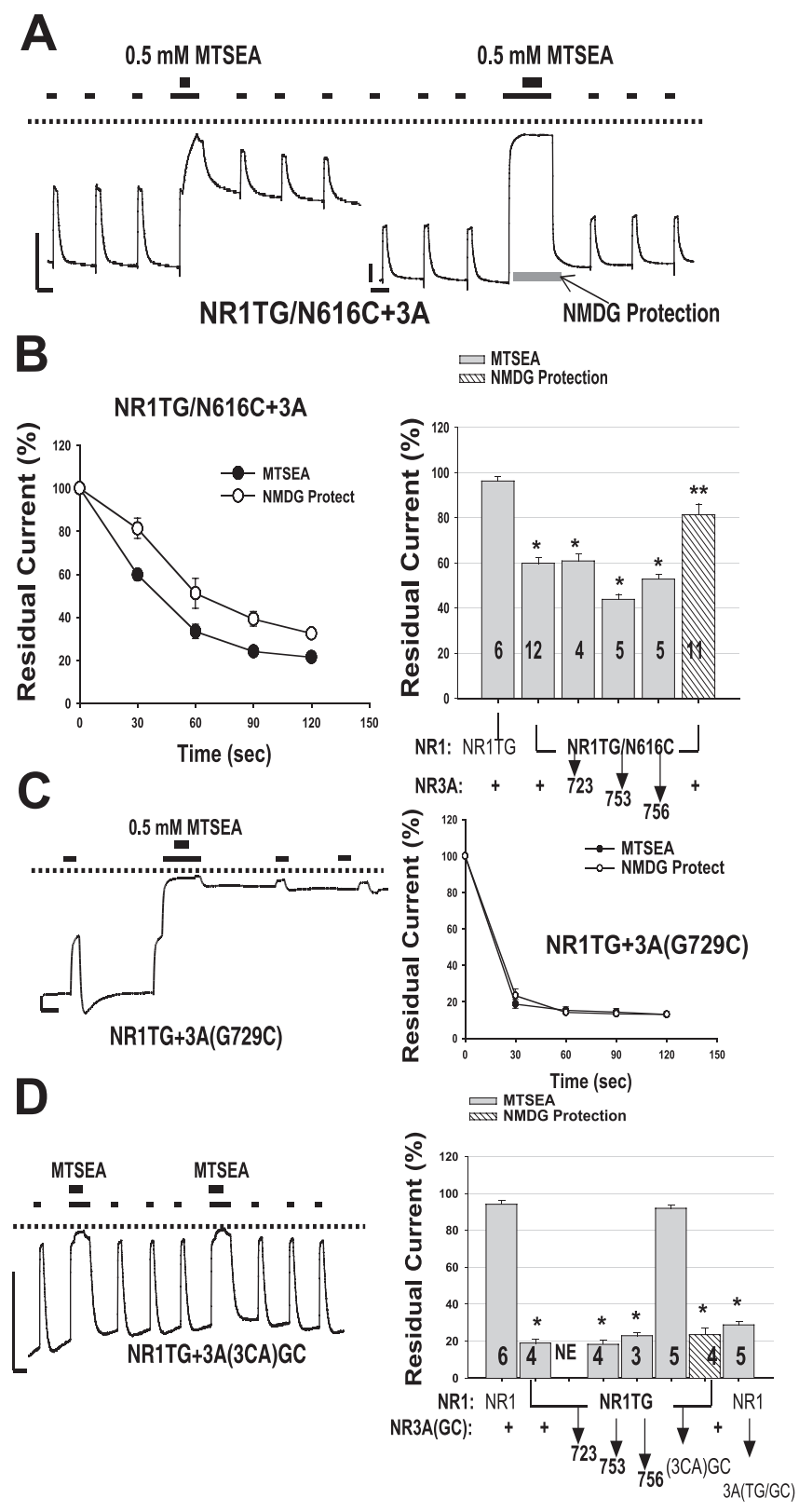

Figure 7. Accessibility pattern of cysteine-substituted mutation at the $\mathrm{N}$-site residue of the M2 segments of NR1 and NR3 after widening the constriction at the outer vestibule of the channel. $\boldsymbol{A}$, Representative recordings showing modulation by external MTS reagents of $\mathrm{NR} 1(\mathrm{~T}+2 \mathrm{G}, \mathrm{N616C)} / \mathrm{NR3}$ A mutant channels (designated NR1TG/N616C + 3A in the figure for brevity). The smaller MTSEA persistently modified the channel. MTSEA modification could be reduced by preexposure to the pore blocker NMDG $(96 \mathrm{~mm})$ (gray tracing at top right). B, NMDG protection assay to test whether a specific amino acid residue lines the channel pore region. Left, Degrees of residual current $\left(=I_{\text {Post-drugs }} / I_{\text {Control }} \times 100 \%\right)$ at $30,60,90$, and 120 s is plotted to show the temporal delay in action of $0.5 \mathrm{~mm}$ MTSEA by the pore blocker NMDG $(96 \mathrm{~mm})$. Right, Residual current after MTSEA modification of various combinations of mutants. The first bar indicates lack of modification by MTSEA $(96.2 \pm 2.2 \%)$ of NR1(T+2G)/NR3A mutants. The second bar shows persistent modification of NR1(T+2G, N616C)/NR3A channels by MTSEA $(59.8 \pm 2.6 \%)$. The third to fifth bars indicate that replacement of endogenous cysteines in the permeation pathway with alanines did not affect modification of the NR1(N616C) residue by MTSEA. The last bar illustrates protection from MTSEA modification by preexposure to NMDG $(81.3 \pm 4.7 \%) . *$ *Statistically significant modification compared with NR1(T+2G)/NR3A channels by an unpaired Student $t$ test. ${ }^{*}$ Significant protection from MTSEA modification on NR1 (T + 2G, N616C)/NR3A mutant channels by NMDG. Error bars indicate SEM. C, Representative recordings of persistent modulation by external MTSEA of currents from NR1 $(T+2 G) /$ NR3A(G729C) (left trace). Right, NMDG protection assay. Degree of modulation at 30, 60, 90, and $120 s$ by MTSEA of NR1(T+2G)/NR3A(G729C) channels is plotted as in $\boldsymbol{B}$; NMDG had no effect on the kinetics of MTSEA modification. D, Persistent modification of currents from
Effect of extracellular MTS reagents on cysteine substitution mutants at the $\mathrm{N}$-site of the pore loop in the M2 segment of NR1/NR3A channels after "widening" the constriction in the M3 segment representing the outer vestibule of the "NMDA" receptor channel

Recall that externally applied MTS reagents did not have access to the $\mathrm{M} 2$ region of NR1/NR3A channels in our initial experiments. Given our subsequent result suggesting a narrow constriction in the outer vestibule of the NR1/NR3A channel, we reasoned that this constriction might limit access to the M2 region, which is located deeper in the channel pore. To test this hypothesis, we designed experiments to physically widen the outer vestibule of the channel to permit access of MTS reagents to the pore loop of the M2 segment. To accomplish this, we mutated the last accessible amino acid residues in the outer vestibule, represented by threonines at the +2 position of M3 segments of both the NR1 and NR3A subunits; these threonines residues were substituted with smaller, more flexible glycine residues, producing NR1 $(\mathrm{T}+2 \mathrm{G})$, designated NR1TG in the figures, and NR3A(T+2G), designated NR3A(TG).

With the NR1 $(T+2 G)$ mutation as backbone, we performed SCAM analysis (as in Fig. 2) to evaluate accessibility from the extracellular side of the membrane of MTS reagent to the critical $\mathrm{N}$-site residues located at the tip of channel selectivity filter. We found that the $\operatorname{NR} 1(T+2 G)$ mutation resulted in a large leak current when coexpressed with wild-type NR3A. Application of $10 \mu \mathrm{M}$ glycine transiently activated a small inward current and subsequently closed the channel (similar to type 3 current in Fig. 3). All NR1/NR3 mutant channels containing the NR1 $(T+2 G)$ mutation displayed similar current characteristics. Externally applied MTSEA did not modify NR1 $(\mathrm{T}+2 \mathrm{G}) / \mathrm{NR} 3 \mathrm{~A}$ channels, indicating that endogenous cysteine residues were not exposed by the $\mathrm{NR} 1(\mathrm{~T}+2 \mathrm{G})$ mutation. We then made an additional N616C mutation in the $\mathrm{NR} 1(\mathrm{~T}+2 \mathrm{G})$ subunit to form the NR1(T+2G, N616C). When coexpressed with NR3A, the currents associated with $\mathrm{NR} 1(\mathrm{~T}+2 \mathrm{G}, \mathrm{N} 616 \mathrm{C})$ were persistently blocked by external MTSEA (Fig. 7A, left-hand trace). This finding suggested that the widened external constriction in the outer vestibule of the channel allowed MTSEA to pass through to reach the $\mathrm{M} 2$ region where it covalently modified the more internal $\mathrm{N}$-site residue. Furthermore, we tested whether this residue could be protected from MTSEA accessibility by the nonspecific and nonpermeable channel pore blocker NMDG (Zarei and Dani, 1995). We found that NMDG indeed provided protection from MTSEA modification (Fig. $7 A$, right-hand trace; $B$, left-hand

$\leftarrow$

NR1 $(T+2 G) / N R 3 A(G 729 C)$ by external MTSEA could be eliminated by replacement of all three endogenous cysteines in the permeation pathway with alanines (designated as NR1TG + NR3A(3CA)GC) (left trace). Right, Residual current after persistent MTSEA modification of various combinations of mutants. The first bar shows lack of modification by MTSEA $(94.1 \pm 2.3 \%)$ on the NR1/3A(G729C), designated NR1/NR3A(GC) for brevity. The second bar shows modification of NR1(T+2G)/NR3A(G729C) channels by MTSEA (18.8 $\pm 2.4 \%)$. The $\mathrm{NR3A}(\mathrm{C} 723 \mathrm{~A}, \mathrm{G} 729 \mathrm{C})$ mutant, replacing the endogenous cysteine at residue 723 in the M2 segment, did not form function channels with NR1 $(T+2 G)$. NE, No expression. The fourth and fifth bars indicate that replacement of the two endogenous cysteines in the permeation pathway formed by the M3 segment with alanines (NR3A C753A and C756A) did not affect modification on the NR3A(G729C) residue. The sixth bar shows that replacement of all three endogenous cysteines in the $M 2$ and $M 3$ segments abrogated modification by MTSEA. The seventh bar shows that preexposure to NMDG did not protect NR1(T + 2G)/NR3A(G729C) from MTSEA modification. The last bar indicates that widening the outer vestibule of the channel by mutating $\mathrm{NR3A}(\mathrm{T}+2 \mathrm{G})$ also permitted modification of the NR3A(G729C) residue by MTSEA (28.6 \pm 1.9\%). *Statistically significant modification compared with NR1/NR3A(G729C) channels by an unpaired Student $t$ test. Calibration: $100 \mathrm{nA}, 30 \mathrm{~s}$. The number in each column represents oocytes tested. 
graph), consistent with the notion that the NR1(N616) residue is indeed located at the channel selectivity filter of NR1/NR3 channel, similar to that described for the M2 segment of the NR1 subunit in classical NR1/NR2 channels (Kuner et al., 1996). To avoid possible confounding effects of the endogenous cysteine residues that line the permeation pathway, we also mutated all three of these cysteines in the M2 and M3 segments of the NR3A subunit to alanines [designated NR3A(C723A), NR3A(C753A), and NR3A(C756A)]. When coexpressed with each of these three NR3A mutants, modification of NR1( $T+2 G, N 6161 C)$ by external MTSEA remained unchanged (Fig. $7 B$, right-hand graph), indicating that the endogenous cysteine residues in the NR3A subunit did not influence this result. Coexpression of the $\mathrm{NR} 1(\mathrm{~T}+2 \mathrm{G}, \mathrm{N} 616 \mathrm{C})$ and NR3A(T+2G) mutants did not form functional channels and therefore could not be tested.

The results of MTS modification of channels containing the NR1(N616C) mutation are summarized in Figure 7B, right-hand graph. Note that the diameter of the head group of the MTSEA molecule is estimated to be $3.6 \AA$ (Sobolevsky et al., 2004), and its overall size is $<5.8 \times 5.8 \times 10 \AA$ in the all-trans configuration (Karlin and Akabas, 1998; Hille, 2001). Considering the fact that none of the cysteine substitutions in the M2 segment of the NR1 or NR3A subunits was accessible to external MTSEA before widening of the outer vestibule of the channel by mutation, the narrow constriction at the outer channel vestibule is probably $<3.6-$ $5.8 \AA$ in diameter.

Next, we studied the N-site equivalent of the NR3A subunit, which is located at the G729 residue and was also not accessible to external MTS reagents before widening of the outer vestibule of the channel (Fig. 1). We used the NR1 $(\mathrm{T}+2 \mathrm{G})$ mutant and SCAM analysis to evaluate accessibility of this site after widening the outer vestibule. The cysteine residue in NR3A(G729C) was persistently modified by external MTSEA when this subunit was coexpressed with the NR1 $(\mathrm{T}+2 \mathrm{G})$ mutant (Fig. 7C, left-hand trace). However, MTSEA modification of the NR1 $(T+2 G) /$ $3 \mathrm{~A}(\mathrm{G} 729 \mathrm{C})$ channel was not protected by the pore blocker, NMDG (Fig. 7C, right-hand graph). This result prompted us to investigate the possible confounding effect of the endogenous cysteine residues that line the permeation pathway. To eliminate this possible confounding effect, we again substituted the three endogenous cysteines in the permeation pathway with alanines. Unfortunately, the resulting NR3A(G729C) mutant with an additional alanine substitution for the endogenous cysteine in the M2 segment, NR3A(C723A, G729C), did not form functional channels with NR1 $(T+2 G)$. Mutations of the other two endogenous cysteines, which are located in the M3 segment of the NR3A subunit $[\mathrm{NR} 3 \mathrm{~A}(\mathrm{C} 753)$ and NR3A(C756)], did not affect the observed modification by MTSEA. However, modification by MTS agents was completely eliminated by removing all three endogenous cysteines in the M2 and M3 segments [designated as the NR3A(3CA) mutant] (Fig. 7D, left-hand trace). This result is consistent with the notion that the $\mathrm{NR} 3 \mathrm{~A}(\mathrm{C} 723)$ residue in the M2 segment is a confounding residue that was susceptible to modification by external MTS reagent in the presence of the NR3A(G729C) mutation. Because the pore blocker NMDG did not protect this modification, these findings also suggest that the $\mathrm{NR} 3 \mathrm{~A}(\mathrm{C} 723)$ residue is positioned close to but not in the lining of the selectivity filter region of NR1/NR3A channels. A likely explanation for these results is that G729 in the NR3A M2 segment, unlike in the NR1(N616) residue, is not exposed and therefore does not contribute to the water-accessible channel filter region in the ligand-bound state. Although other possibilities may be envisaged, together, the data suggest that this is the most likely scenario. Because of the complex interactions of mutations in the M2 segment of the NR3A subunit, the exact configuration of this segment will require additional structural studies, which are currently in progress.

\section{Discussion}

NR1 and NR3 M3 segments form a narrow constriction in the outer vestibule of the NMDA receptor channel

M3 segments have been proposed to represent the transduction element of NR1/NR2 NMDARs, coupling ligand binding to channel opening (Jones et al., 2002). Here, we found that cysteine substitutions in this region frequently affect gating kinetics of NR1/NR3 channels. For example, substitution at the $+1,+4$, $+6,+9$, or +10 positions of either subunit reduced or eliminated the initial rapid decay of the response. Mutations at the +4 and +6 positions dramatically reduced current amplitude. Changes in gating behavior similar to those produced by cysteine substitutions in the M3 segments of NR1/NR3A channels were not found in NR1/NR2 channels (Beck et al., 1999), suggesting a different role for $\mathrm{M} 3$ in the gating mechanism of NR1/NR3A channels.

In this study, we derived structural information about the NR3 subunit from SCAM. Interpretation of these results is predicated on the assumptions that MTS reagents react predominantly with exposed cysteine residues and most cysteine substitutions do not significantly alter channel structure (Karlin and Akabas, 1998). Because the M3 segment is essential for gating, cysteine substitution of single residues in the M3 segment frequently changed gating kinetics of NR1/NR3 channels. Our study therefore focused on information inferred from accessibility of the whole M3 region rather than information on a particular amino acid residue, which may prove inaccurate.

In general, the MTSEA accessibility pattern of the M3 segment was similar for NR1 and NR3A subunits. Going from the extracellular to the intracellular side of the membrane, the last residue accessible to external MTS reagents in the M3 segment is a threonine at the +2 position of both the NR1 and NR3A subunits. Considering cysteine mutations of the $\mathrm{T}+2$ position, modification by either MTSEA or MTSET decreased the current amplitude of NR1 $(\mathrm{T}+2 \mathrm{C}) / \mathrm{NR} 3 \mathrm{~A}$ channels. MTSEA modification of NR1/NR3A $(T+2 C)$ channels also decreased the peak current amplitude, but this effect was masked by potentiation of the sustained component of the current (Fig. $4 B$ ). The reaction rate of MTSEA with these threonines on either subunit was slow, even in the open state. Moreover, the NR3A $(T+2 \mathrm{C})$ residue was not accessible to the larger MTSET reagent. MTSET accessibility of the NR1 M3 segment in NR1/NR3 channels, regardless of the charge of the residues, was also more limited than MTSEA, even in the fully open state. These results are consistent with the notion that threonines at the +2 position of both subunits form a narrow constriction at the external channel vestibule. The addition of an ethylamine group from MTSEA at this narrow constriction blocked the channel.

\section{The M3 segment of NR3A lacks state-dependent molecular rearrangement and forms a rigid structure modifying the channel vestibule}

Based on state-dependent changes in the rate of modification of exposed cysteines by MTSEA, the walls lining the pore of the outer vestibule of NR1/NR2 channels is believed to constrict during channel closure (Sobolevsky et al., 2002a). Regarding NR1/ NR3A channels, we found that added glycine opens these channels and accelerates MTSEA modification rates at the M3 segment of NR1 but not the NR3A subunits. The absence of 
A

NR1/NR2

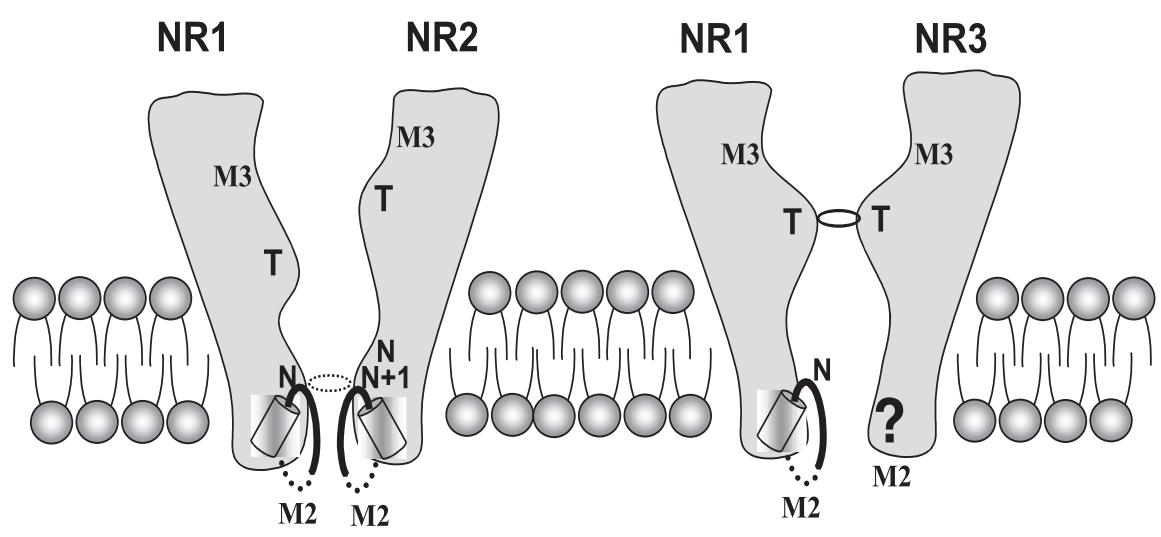

B

\section{NR1/NR3}

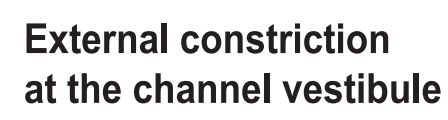

Figure 8. Schematic model for the permeation pathway of "NMDAR" channels. $\boldsymbol{A}$, Model of the permeation pathway proposed for NR1/NR2 NMDAR channels. The M3 segments from the two subunits are staggered relative to each other in the vertical axis of the channel. The $\mathrm{N}$-site residue in the $\mathrm{M} 2$ segment of NR1 and the $\mathrm{N}+1$ site residue in NR2 constitute the selectivity filter of the channel pore (for review, see Wollmuth and Sobolevsky, 2004). B , Proposed model for NR1/NR3A. NR3 subunits replace NR2 subunits to form a symmetrical glycine-activated channel. The NR3A M3 segment forms a nonmobile, rigid structure that participates in the formation of the outer vestibule of the channel. This rigid NR3 M3 domain results in a ring of threonine residues (T), composed of NR3A threonines and the homologous NR1 threonines. Because of the lack of motion of the NR3 subunit at this critical region during channel gating, the ring of threonines forms a constriction in the outer vestibule of the NMDA receptor channel, external to the known selectivity filter in pore loop of the $\mathrm{M} 2$ region (labeled as $\mathrm{N}$-site asparagines). We speculate that this external constriction limits ionic flow (resulting in smaller conductance), disrupts divalent hopping relays (producing less calcium permeability), and changes the sensitivity to divalent block (rendering the channel less sensitive to magnesium block). The conformation and role of the M2 segment from NR3A subunits remain to be elucidated (indicated with a question mark).

state-dependent changes in modification rates of the NR3A M3 segment with MTSEA cannot be explained by membrane permeability because charged MTSET and zinc also reacted with this M3 segment.

A recent paper by Yao and Mayer (2006) showed that the affinity of NR3A for glycine is $\sim 40 \mathrm{~nm}$. The lack of agonistdependent changes observed for MTS modification rates in our study could result from trace glycine contaminating our solutions, producing a fully liganded state on the NR3 subunit. This alternative explanation, although possible, is also unlikely for the following reasons. First, for NR1/NR2A receptors, the modification rate of residues at the +6 position in the M3 region was accelerated by ligand binding to both NR1 and NR2 (Jones et al., 2002). By analogy, the modification rate of residues internal to NR3A $(A+6 C)$ by external MTSEA should have been accelerated by exogenous glycine because the NR1 binding sites were not occupied $\left(K_{\mathrm{d}}=26.4 \mu \mathrm{M}\right)$ (Yao and Mayer, 2006). Second, the slow reaction rate of external MTSEA with the $T+2$ residue of the NR3A subunit and its lack of modification by larger MTSET support the conclusion that this residue is in a constricted location. Third, $10 \mu \mathrm{M}$ glycine closed NR1/NR3A(A+3C) channels $>60-70 \%$, and there was no detectible difference in modification rates in either the open or the closed state of this channel. Therefore, trace amounts of glycine, if present, did not affect the interpretation of our experiments. We thus conclude that the NR3A M3 segment does not undergo molecular rearrangement during gating triggered by exogenous glycine, suggesting a rigid and nonflexible structure. The alternative explanation of this re- sult would be that the NR3A M3 segment is locked permanently in an "activated" conformation and the movement of the NR1 M3 segments is sufficient for channel opening.

Zinc coordination suggests symmetrical alignment of the outer vestibule of the NR1/NR3A channel

$\mathrm{Zn}^{2+}$ coordination has been used to indicate the close proximity of cysteine substitutions at the N-site of both NR1 and NR2 subunits in NR1/NR2 channels (Amar et al., 2001). We also found that zinc persistently inhibited the $\mathrm{NR} 1(\mathrm{~T}+2 \mathrm{C}) /$ $\mathrm{NR} 3 \mathrm{~A}(\mathrm{~T}+2 \mathrm{C})$ current by coordinating the vicinal cysteine substitutions at the +2 position of the $\mathrm{M} 3$ segments. NR1 $(\mathrm{A}+3 \mathrm{C})$ did not result in zinc coordination when coexpressed with NR3A $(\mathrm{T}+2 \mathrm{C})$. These results support the notion that the M3 segments of both subunits are symmetrically aligned. The relatively high concentration of zinc needed in this experiment simply indicates that the cysteine substitutions of the neighboring threonines are not in a tetrahedral configuration for chelating the zinc (Holm et al., 1996).

\section{The $\mathrm{N}$-site residue in the NR3A M2 segment does not contribute to the selectivity filter}

In conventional NMDARs, asparagines at the $\mathrm{N}$-site of NR1 and the $\mathrm{N}+1$ site of NR2 form the selectivity filter in a reentrant pore loop (Kuner et al., 1996), which controls $\mathrm{Ca}^{2+}$ permeability and $\mathrm{Mg}^{2+}$ block (for review, see Dingledine et al., 1999). After agonist activation, MTSEA can reach $\mathrm{N}$-site residues at the narrowest point of constriction $(<5.5 \AA$ ) (Villarroel et al., 1995) in either the NR1 or NR2 subunit. In contrast, none of the cysteine substitutions in the M2 segment of either NR1 or NR3A is accessible to external MTSEA. This result is consistent with our finding of a narrow constriction that is located in the outer vestibule, external to the selectivity filter region, and thus precludes passage of MTSEA.

After widening the external constriction by substituting the last accessible threonine at the +2 position of the NR1 M3 segment for a smaller glycine residue $[\mathrm{NR} 1(\mathrm{~T}+2 \mathrm{G})]$, the NR1 N-site residue (N616) became accessible to external MTSEA. This reaction was protected by the pore blocker NMDG, indicating that the NR1(N616) contributes to the channel filter of the NR1/ NR3A channel similar to the NR1/NR2 channel. In contrast, after widening the outer vestibule and eliminating endogenous cysteines in the permeation pathway, the $\mathrm{N}$-site equivalent residue (G729) in the NR3A subunit did not appear to react with MTSEA in the ligand-bound state. This apparent lack of reaction with MTSEA suggests that NR3A(G729) is not part of the channel filter. Alternatively, if NR3A(G729C) had a functionally silent reaction with MTSEA, this result would indicate that $\mathrm{NR} 3 \mathrm{~A}(\mathrm{G} 729)$ is not an important part of the pore, because its reaction with MTSEA did not produce any visible change in ionic current amplitude. This result will also explain the lack of functional ion channel expression when the pore domain of NR1 
subunit was swapped with the M2 segment of NR3A (Villmann et al., 1999).

\section{Structural implications for NR3A-containing channels}

Model A in Figure 8 illustrates a possible configuration of the permeation pathway of NR1/NR2 channels as reviewed by Wollmuth and Sobolevsky (2004). In NR1/NR2 NMDARs, residues in the M3 segment of NR2 are about 4 aa more external than homologous positions in NR1. Asparagines at the NR1 N-site and the NR2 $\mathrm{N}+1$ site form the selectivity filter.

In contrast, based on our SCAM analysis, model B in Figure 8 shows that NR1 and NR3A form a symmetrical configuration in the M3 segment comprising the outer vestibule with homologous residues lying at the same level along the vertical axis of the channel. This symmetrical NR1/NR3A channel is similar to AMPAand kainate-gated receptor channels. Furthermore, our evidence suggests that the TYTANLAAV motif of the NR3A M3 segment forms a rigid structure that contributes to the outer vestibule of the channel, yet lacks state-dependent (i.e., open-closed state) conformational changes. Incorporation of such a nonflexible structure would be expected to modify the properties of NR1/ NR2/NR3A and NR1/NR3A channels. Most importantly, a ring of polar threonine residues at the +2 position in both NR1 and NR3A subunits form a "constriction" barrier external to the selectivity filter and restrict access of MTS reagents to the M2 region. This model of external constriction is further supported by the accessibility of NR1(N616C) after physically widening the outer vestibule of the channel by mutations. Additionally, recent findings indicate that conventional NMDARs display high $\mathrm{Ca}^{2+}$ permeability because of the DRPEER motif and the $\mathrm{N}+4$ residue in the M3 segment, which form a chain of calcium-binding sites to facilitate $\mathrm{Ca}^{2+}$ flux (Watanabe et al., 2002). The external constriction of the NR1/NR3 channel would be expected to disturb these "hopping" relay stations and further reduce the calcium permeability.

\section{References}

Amar M, Perin-Dureau F, Neyton J (2001) High-affinity Zn block in recombinant $N$-methyl-D-aspartate receptors with cysteine substitutions at the $\mathrm{Q} / \mathrm{R} / \mathrm{N}$ site. Biophys J 81:107-116.

Beck C, Wollmuth LP, Seeburg PH, Sakmann B, Kuner T (1999) NMDAR channel segments forming the extracellular vestibule inferred from the accessibility of substituted cysteines. Neuron 22:559-570.

Chatterton JE, Awobuluyi M, Premkumar LS, Takahashi H, Talantova M, Shin Y, Cui J, Tu S, Sevarino KA, Nakanishi N, Tong G, Lipton SA, Zhang D (2002) Excitatory glycine receptors containing the NR3 family of NMDA receptor subunits. Nature 415:793-798.

Chen PE, Geballe MT, Stansfeld PJ, Johnston AR, Yuan H, Jacob AL, Snyder JP, Traynelis SF, Wyllie DJ (2005) Structural features of the glutamate binding site in recombinant NR1/NR2A $N$-methyl-D-aspartate receptors determined by site-directed mutagenesis and molecular modeling. Mol Pharmacol 67:1470-1484.

Choi DW (1998) Antagonizing excitotoxicity: a therapeutic strategy for stroke? Mt Sinai J Med 65:133-138.

Das S, Sasaki YF, Rothe T, Premkumar LS, Takasu M, Crandall JE, Dikkes P, Connor DA, Rayudu PV, Cheung W, Chen H-SV, Lipton SA, Nakanishi N (1998) Increased NMDA current and spine density in mice lacking the NMDA receptor subunit NR3A. Nature 393:377-381.

Dingledine R, Borges K, Bowie D, Traynelis SF (1999) The glutamate receptor ion channels. Pharmacol Rev 51:7-61.

Hille B (2001) Ion channel of excitable membranes. Sunderland, MA: Sinauer.

Holm RH, Kennepohl P, Solomon EI (1996) Structural and functional aspects of metal sites in biology. Chem Rev 96:2239-2314.

Jones KS, VanDongen HM, VanDongen AM (2002) The NMDA receptor
M3 segment is a conserved transduction element coupling ligand binding to channel opening. J Neurosci 22:2044-2053.

Karlin A, Akabas MH (1998) Substituted-cysteine accessibility method. Methods Enzymol 293:123-145.

Kuner T, Wollmuth LP, Karlin A, Seeburg PH, Sakmann B (1996) Structure of the NMDA receptor channel M2 segment inferred from the accessibility of substituted cysteines. Neuron 17:343-352.

Lipton SA, Rosenberg PA (1994) Mechanisms of disease: excitatory amino acids as a final common pathway for neurologic disorders. N Engl J Med 330:613-622.

Liu Y, Holmgren M, Jurman ME, Yellen G (1997) Gated access to the pore of a voltage-dependent $\mathrm{K}^{+}$channel. Neuron 19:175-184.

Matsuda K, Kamiya Y, Matsuda S, Yuzaki M (2002) Cloning and characterization of a novel NMDA receptor subunit NR3B: a dominant subunit that reduces calcium permeability. Brain Res Mol Brain Res 100:43-52.

Mayer ML, Westbrook GL, Guthrie PB (1984) Voltage-dependent block by $\mathrm{Mg}^{2+}$ of NMDA responses in spinal cord neurons. Nature 309:261-263.

Nakanishi S (1992) Molecular diversity of glutamate receptors and implications for brain function. Science 258:597-603.

Nishi M, Hinds H, Lu HP, Kawata M, Hayashi Y (2001) Motoneuronspecific expression of NR3B, a novel NMDA-type glutamate receptor subunit that works in a dominant-negative manner. J Neurosci 21:RC185(1-6).

Nowak L, Bregestovski P, Ascher P, Herbet A, Prochiantz A (1984) Magnesium gates glutamate-activated channels in mouse central neurons. Nature 307:462-465.

Paoletti P, Ascher P, Neyton J (1997) High-affinity zinc inhibition of NMDA NR1-NR2A receptors. J Neurosci 17:5711-5725.

Piña-Crespo JC, Heinemann SF (2004) Physiological and pharmacological properties of recombinant NR3-type receptors expressed in mammalian cells. Soc Neurosci Abstr 30:957.1.

Sasaki YF, Rothe T, Premkumar LS, Das S, Cui J, Talantova MV, Wong HK, Gong X, Chan SF, Zhang D, Nakanishi N, Sucher NJ, Lipton SA (2002) Characterization and comparison of the NR3A subunit of the NMDA receptor in recombinant systems and primary cortical neurons. J Neurophysiol 87:2052-2063.

Sobolevsky AI, Beck C, Wollmuth LP (2002a) Molecular rearrangements of the extracellular vestibule in NMDAR channels during gating. Neuron 33:75-85.

Sobolevsky AI, Rooney L, Wollmuth LP (2002b) Staggering of subunits in NMDAR channels. Biophys J 83:3304-3314.

Sobolevsky AI, Yelshansky MV, Wollmuth LP (2004) The outer pore of the glutamate receptor channel has 2 -fold rotational symmetry. Neuron 41:367-378.

Sucher NJ, Akbarian S, Chi CL, Leclerc CL, Awobuluyi M, Deitcher DL, Wu MK, Yuan JP, Jones EG, Lipton SA (1995) Developmental and regional expression pattern of a novel NMDA receptor-like subunit (NMDAR-L) in the rodent brain. J Neurosci 15:6509-6520.

Sullivan JM, Traynelis SF, Chen H-SV, Escobar W, Heinemann SF, Lipton SA (1994) Identification of two cysteine residues that are required for redox modulation of the NMDA subtype of glutamate receptor. Neuron 13:929-936.

Villarroel A, Burnashev N, Sakmann B (1995) Dimensions of the narrow portion of a recombinant NMDA receptor channel. Biophys J 68:866-875.

Villmann C, Strutz N, Morth T, Hollmann M (1999) Investigation by ion channel domain transplantation of rat glutamate receptor subunits, orphan receptors and a putative NMDA receptor subunit. Eur J Neurosci 11:1765-1778.

Watanabe J, Beck C, Kuner T, Premkumar LS, Wollmuth LP (2002) DRPEER: a motif in the extracellular vestibule conferring high $\mathrm{Ca}^{2+}$ flux rates in NMDA receptor channels. J Neurosci 22:10209-10216.

Wollmuth LP, Sobolevsky AI (2004) Structure and gating of the glutamate receptor ion channel. Trends Neurosci 27:321-328.

Yao Y, Mayer ML (2006) Characterization of a soluble ligand binding domain of the NMDA receptor regulatory subunit NR3A. J Neurosci 26:4559-4566.

Zarei MM, Dani JA (1995) Structural basis for explaining open-channel blockade of the NMDA receptor. J Neurosci 15:1446-1454. 